\title{
Delayed interval twin delivery of a fetus with a favourable neonatal outcome after a preterm delivery of the first twin: a case report
}

\author{
Nitin P. PaiDhungat, Tejaswi D. Kamble, Alka C. Bapat*
}

Department of Obstetrics and Gynecology, Bombay Hospital Institute of Medical Sciences, Mumbai, Maharashtra, India

Received: 26 July 2018

Accepted: 28 August 2018

*Correspondence:

Dr. Alka C. Bapat,

E-mail: bapat.alka@gmail.com

Copyright: (c) the author(s), publisher and licensee Medip Academy. This is an open-access article distributed under the terms of the Creative Commons Attribution Non-Commercial License, which permits unrestricted non-commercial use, distribution, and reproduction in any medium, provided the original work is properly cited.

\begin{abstract}
Assisted reproductive techniques have proved to be a boon for infertile couples. With advent of newer techniques, the incidence of successful multiple pregnancies has also risen. Considering the emotional and financial aspects of the treatment and the risk of preterm delivery in such cases, our intent is not only to salvage one of the twins in case of unfortunate preterm delivery of the other but also to deliver a viable second twin with better chance of survival and favourable neonatal outcome. The current case describes a 34-year woman with previous 2 failed IVF conceptions, on external progesterone support, carrying a twin gestation in preterm labour. Upon the inadvertent delivery of the first twin, a cervical cerclage was done, and she was given conservative management, including bed rest and head low position in view of short cervix, with an aim to delay the delivery of the other. An interval of 66 days was achieved with surgical as well as medical management, following which a healthy second twin was born.
\end{abstract}

Keywords: Cervical cerclage, Interval twin, Multiple pregnancy

\section{INTRODUCTION}

With advancement in assisted reproductive technology and its increased use in treatment of infertility, the incidence of multiple pregnancies has risen. Multiple pregnancies are associated with a higher risk of preterm delivery at a significantly earlier gestational age than singletons, resulting in infant morbidity and mortality.

In present case, our patient, a prospective mother of a twin, was given progesterone support. She visited the emergency department at around 21-22 weeks with complaints of spotting and leaking per vaginum. She was advised bed rest and head low position in view of short cervix, in spite of OS tightening, which she could not follow due to social reasons. In pregnancies presenting with extreme preterm labour or rupture of the membranes, significant prolongation of gestation and hence increase in fetal weight is likely to improve fetal outcome. For this reason, it can be tried to stop labour after birth of the first fetus. In one such case of twin pregnancy, the unavoidable birth of one premature neonate has led our efforts to aim for the delayed interval delivery for the other twin, keeping in mind the psychological aspects attached with pregnancy.

The role of prolonged bed rest, cervical cerclage, tocolysis, antibiotics and corticosteroids has been a subject of frequent debate. It is thus important to find any parameters that can predict the length of the latency period, the risk for both mother and fetus, and the outcome of these pregnancies. ${ }^{1-3}$ The aim of this case report is to add our experience to the currently limited literature regarding the best treatment of this unique obstetrical problem. 


\section{CASE REPORT}

A 34-year-old G2 P0 A1 L0, with previous 2 failed IVF conceptions, with 21.5 weeks by dates, 21.2 weeks by scan (A); 21.6 weeks by scan (B), presented in the emergency department with complaints spotting and leaking per vaginum and regular uterine contractions. The heart sounds of the first twin couldn't be localised, whereas those of the second twin were heard and regular. After evaluation of the patient, the spontaneous expulsion of the first twin was undertaken in the Operating room, cord was clamped and cut end ligated high with Polyglactin no.1 round body suture material, with both Placentae being low lying covering the internal os. Cervical Cerclage was done to prevent the delivery of the second twin.

Patient was given complete bed rest, bedside toilet facilities and head low position. Antibiotic coverage with Injectable (Cefoperazone + Sulbactam) $1.5 \mathrm{~g}$ twice daily for 5 days, Metronidazole $(500 \mathrm{mg})$ thrice a day for 3 days was started, followed by oral erythromycin ( 250 $\mathrm{mg}$ ) four times a day after taking a high vaginal swab. Patient was catheterised for some days post procedure with intermittent clamping. Tocolysis was started with $\mathrm{MgSO} 4(5 \mathrm{mg}$ in $500 \mathrm{cc} \mathrm{NS}$, at $80 \mathrm{cc}$ per hour) and supplemented with nifedipine retard preparation $(20 \mathrm{mg})$ to avoid setting of preterm labour. Five doses of Betamethasone $(12 \mathrm{mg}$ ) Injection, 2 per week - 24 hours apart were given. $\mathrm{MgSO} 4$ tocolysis was stopped after 24 hours and was repeated only when examination revealed mild uterine contractions during 29th week of gestation. Iron, Calcium and Protein supplements were continued along with Aspirin (75 mg) once daily, which was started in view of her obstetric history. Injectable Progesterone (Dydrogesterone $200 \mathrm{mg}$ ) IM was given every alternate day, along with oral Progesterone (micronized progesterone $200 \mathrm{mg}$, twice a day) and Estradiol valerate ( $2 \mathrm{mg}$, twice a day) throughout the pregnancy. Weekly CRP, coagulation profile, CBC were monitored, to ensure no impaired coagulation or raised counts. Antibiotic ointment locally and Clotrimazole (antifungal) vaginal pessary were given for complaints of itching and minimal non-foul-smelling vaginal discharge. Obstetric ultrasound was done once in 2-3 weeks to check cervical length, fetal wellbeing and for placental position. The last scan at 28 weeks, showed the placentae to be low lying, covering the internal os and baby weight exceeding $1 \mathrm{~kg}$.

On day 66 of the Cervical Cerclage surgery, at gestational age of 31 weeks, patient passed large clots and started getting uterine contractions. A decision to terminate the pregnancy with lower segment caesarean section was taken. A female child with a birth weight of $1360 \mathrm{~g}$ was born, with the baby crying at 30 seconds after birth with stimulation. Two placentae were born, of which one was fibrous and calcified with necrotic umbilical cord. A bucket handle tear of the cervix was noticed post-delivery after the cerclage stitch removal. Post-operative recovery of the mother needed special physiotherapy assistance for lower limb mobilisation due to loss of muscle strength, however the rest course was uneventful. The neonate was admitted in the NICU for 1 month and discharged at weight of $1500 \mathrm{~g}$ in stable condition. The baby was followed up subsequently for eye screening and milestones which were satisfactory till date.

\section{DISCUSSION}

Multiple pregnancies are associated with a higher risk of preterm delivery and this at a significantly earlier gestational age than singletons, resulting in infant morbidity and mortality. ${ }^{4}$ Very few cases of intentional delayed delivery of the second twin have been reported in literature. ${ }^{5}$ There is no set protocol regarding the best management of these pregnancies. The role of prolonged bed rest, cervical cerclage, tocolysis, antibiotics and corticosteroids has been a subject of frequent debate. The management described in this case report may thus not be the only adequate treatment. Authors feel there is a need of discussion and evolution of a standard protocol for management of delayed interval twin delivery.

Antenatal complications are common during multiple pregnancies and the number of the embryos influences both their frequency and the gestational age at which they appear. The main problem appears to be preterm labour and preterm premature rupture of the membranes, with one condition often leading to the other. A possible reason for the premature rupture of the membranes could be an ascending infection from the vagina or the cervix into the uterine cavity. This tends to occur more often when there is already some degree of cervical dilatation, as in cases of cervical incompetence or increased intrauterine pressure. Infection and rupture of the membranes can lead to uterine contractions and subsequent delivery. ${ }^{2}$

Cervical cerclage, if decided, is advised to be done in aseptic conditions, during the first two hours after the birth of the first fetus and when there is no evidence of infection. Both the expediency and the effectiveness of this action is a matter of dispute among obstetricians. To avoid ascending infection, the cord of the first-born twin should be ligated, with an absorbable suture, as close to the cervix as possible, under aseptic conditions. It is recommended that women should stay in bed, for the rest of their pregnancy, although there are rare reports where patients were permitted to leave hospital under medical supervision. ${ }^{5}$ Suppression of premature contractions can be achieved with tocolytics like beta receptor agonists, magnesium sulphate, oxytocin-receptor inhibitor or nonsteroidal anti-inflammatory drugs. Tocolysis may be used as a precautionary measure after the birth of the first twin, or only later during uterus contractions, but never in the presence of a well-established chorioamnionitis.

After premature rupture of the membranes, infection could be suspected on the basis of a rise of the temperature, of the white blood cell count and of the CReactive Protein (CRP). CRP is considered as a good 
prognostic index for incipient chorioamnionitis. ${ }^{1,5}$ Antibiotics like penicillin or cephalosporins must be provided immediately after the birth of the first child. Again, authors find no unanimity about their administration. Corticosteroids can be administered in the absence of chorioamnionitis, with beneficial results.

In reviewing the literature, the aim was to summarize the overall experience of the management and outcome of the retained fetus, in cases of twin pregnancies, after delivery of the first. In most cases the outcome of the second twin was favourable, in contrast to the bad outcome of the first twin. ${ }^{6}$ Survival of the first born was clearly linked to its gestational age and birth-weight. The survival of the second born was dependent upon a number of factors, including the delivery interval between the first and second twin and the presence of obstetric problems appearing during the latency period; the longer the interval, the greater was the chance for surviving. Likewise, the absence of significant obstetric problems in the latency period also improved survival.,

Modern management procedures including tocolytics, corticosteroids for lung maturation, antibiotics and cervical cerclage are important in the overall success of treatment in a retained twin. No statistically significant difference was found, although both tocolytics and cerclage appear to prolong the mean delivery interval. ${ }^{2}$ There are no clear indications for the use of prophylactic cervical cerclage and it doesn't appear to improve the survival of the second twin. ${ }^{7}$ The use of antibiotics is widely practiced, but its use after isolation of a specific pathogen in cervicovaginal secretions is preferred. Vaginal examinations should be avoided; however, the length and dilatation of the cervix should be followed ultrasonographically. Monitoring should be carried out and limited to a weekly full blood count, coagulation profile. Even a large placental mass can be retained in the uterus and produce no demonstrable clinical symptoms. ${ }^{2}$

Attempts for delayed interval delivery should be made when the first neonate is born before the 24th week of gestation, aiming the prolongation of second twin's delivery until the $28^{\text {th }}$ to $32^{\text {nd }}$ week..$^{1,8-11}$ Although there is difference of opinion regarding use of cervical cerclage, it may be considered along with modern management procedures. $^{12}$ Decision for the continuation of the pregnancy after the $32^{\text {nd }}$ week has to be taken with circumspection, and risks versus the benefits for continuation of the pregnancy need to be analysed.

\section{CONCLUSION}

In the analysis of this case, there was no evidence that retention of placenta caused disseminated intravascular coagulation and did not seem initiate an intra-uterine infection. Fever and hemorrhage were relative contraindications for our decision about the use of tocolysis. Administration of indomethacin could impede the diagnosis of chorioamnionitis, hence only limited use was done. In present case the pregnancy was terminated with an emergency cesarean section due to onset of labour and ante partum hemorrhage. Authors applied the aforementioned surgical and pharmaceutical treatment with successful outcome. More studies are required for developing a generalised management process especially in terms of performing cerclage in view of diverse opinions.

Delayed interval pregnancy appears to be safe for the mother and can salvage the fetus. Hence there is a need for documenting more cases to establish a protocol for management of these patients.

\section{ACKNOWLEDGMENTS}

Authors thank Dr. S Goyal, Head, Department of Obstetrics and Gynecology, Bombay Hospital Institute of Medical Sciences, Mumbai, Maharashtra, India for support and encouragement.

Funding: No funding sources

Conflict of interest: None declared

Ethical approval: Not required

\section{REFERENCES}

1. Arias F. Delayed delivery of multifetal pregnancies with premature rupture of membranes in the second trimester. Am J Obstet Gynecol. 1994;170:1233-7.

2. de Jong MW, van Lingen RA, Wildschut J, van Eijck J. Delayed interval delivery of two remaining fetuses in Quintuplet Pregnancy after embryo reduction: Report and review of the literature. Acta Genet Med Gemellol. 1992;41:49-52.

3. Farkouh LJ, Sabin ED, Heyborne KD, Lindsay GL, Porreco RP. Delayed interval delivery: Extended Series from a single maternal-fetal medicine practice. Am J Obstet Gynecol. 2000;183(6):1499-503.

4. Gardner MO, Goldenberg RL, Cliver SP, Tucker JM, Nelson KG, Copper RL. The origin and outcome of preterm twin pregnancies. Obstet Gynecol. 1995;85(4):553-7.

5. Wittmann BK, Farguhanson D, Wong JP, Baldwin V, Wadsworth LD, Ellit L. Delayed delivery of second twin: report of four cases and review of the literature. Obstet Gynecol. 1992;79:260-3.

6. Kalchbrenner MA, Weisenborn EJ, Chyu JK, Kaufman HK, Losure TA. Delayed delivery of multiple gestations: maternal and neonatal outcomes. Am J Obstet Gynecol. 1998;179:1145-9.

7. Weemhoff M, van Meir CA, Walther FJ, Twaalfhoven FC. Delayed birth of the second child in multiple gestation. Ned Tijdschr Geneeskd. 2001;145:1377-80.

8. Poeschmann PP, Oppen CA, Bruinse HW. Delayed interval delivery in multiple pregnancies: report of three cases and review of the literature. Obstet Gynecol Surv. 1992;47:139-41. 
9. Tzafettas JM, Farmakides G, Delkos D, Kalogiros G, Gkoutzioulis F, Psarra A, et al. Asynchronous delivery of twins and triplets with a delivery interval ranging from 48 hours to 19 weeks. Clin Exp Obstet Gynecol. 2004;31:53-5.

10. Klearhou N, Mamopoulos A, Pepes S, Daniilidis A, Rousso D, Karagiannis V. Delayed interval delivery in twin pregnancy: A case report. Hippokratia. 2007;11(1):44-6.

11. Padilla-Iserte P, Vila-Vives JM, Ferri B, GómezPortero R, Diago V, Perales-Marín A. Delayed interval delivery of the second twin: obstetric management, neonatal outcomes, and 2-year followup. J Obstet Gynaecol India. 2014;64:344-8.
12. Cozzolino M, Seravalli V, Masini G, Pasquini L, Di Tommaso M. Delayed interval delivery in dichorionic twin pregnancies: A single center experience. Ochsner J. 2015;15(3):248-50.

Cite this article as: PaiDhungat NP, Kamble TD, Bapat AC. Delayed interval twin delivery of a fetus with a favourable neonatal outcome after a preterm delivery of the first twin: a case report. Int J Reprod Contracept Obstet Gynecol 2018;7:4309-12. 